\title{
Using Isophotes and Shadows to Interactively Model Normal and Height Fields
}

\author{
Qiuying Xu ${ }^{\mathrm{a}}$, Songrun $\mathrm{Liu}^{\mathrm{b}}$, Yotam Gingold ${ }^{\mathrm{b}}$, Karan Singh ${ }^{\mathrm{a}}$ \\ ${ }^{a}$ University of Toronto \\ ${ }^{b}$ George Mason University
}

\begin{abstract}
We introduce an interactive modeling tool for designing (a) a smooth 3D normal field from the isophotes of a discretely shaded 2D image and (b) lifting the normal field into a smooth height field given a cast shadow. Block or cartoon shading is a visual style in which artists depict a smoothly shaded 3D object using a small number of discrete brightness values, manifested as regions or bands of constant color. In our approach, artists trace isophotes, or curves of constant brightness, along the boundaries between constant color bands. Our algorithm first estimates light directions and computes 3D normals along the object silhouette and at intersections between isophotes from different light sources. We then propagate these $3 \mathrm{D}$ normals smoothly along isophotes, and subsequently throughout the interior of the shape. We describe our user interface for editing isophotes and correcting unintended normals produced by our algorithm. We also describe a technique for lifting the generated normal field into a height field given the boundary of the shadow cast by the object. We validate our approach with a perceptual experiment and comparisons to ground truth data. Finally, we present a set of 3D renderings created using our interface.
\end{abstract}

Keywords: sketching, non-photorealistic rendering, isophotes

2010 MSC: $65 \mathrm{D} 18$

\section{Introduction}

2 Cartoon or toon shading is a visual style for computer${ }_{3}$ generated artwork that portrays the brightness of a shaded sur${ }_{4}$ face by binning or discretizing the brightness values into a small 5 number of bins (Figure 1). The smooth brightness value at a ${ }_{6}$ point on a Lambertian surface with normal $n$ and light direction ${ }_{7} l$ can be computed as $k=\max (n \cdot l, 0)$. In toon shading, $k$ is 8 rounded to one of $b$ bins: $\left\lfloor b k+\frac{1}{2}\right\rfloor$. Toon shading is so-called ${ }_{9}$ because it resembles hand-drawn cartoons. 2D artists employ 10 toon-style shading, or other forms of discrete tone shading, such 11 as hatching at discrete tone levels [1] or blocking large areas 12 of light and shade [2], as an effective way to quickly convey ${ }_{13} 3 \mathrm{D}$ relief information (Figure 1). While discretely shaded line ${ }_{14}$ drawings are compelling in their own right, artists often refine 15 them into smooth-shaded realistic imagery called presentation ${ }_{16}$ renderings, when communicating their designs [3, 4]. Creating 17 these smooth 3D renderings, often using a variety of colors, 18 materials and lighting is tedious, labor intensive and error prone. ${ }_{19}$ Inspired by CrossShade [5], which addresses the presentation 20 rendering problem for cross-sectional line drawings, we present ${ }_{21}$ an interactive modeling tool to assist artists with inverse toon 22 shading.

23 Inverse toon shading is the transformation of a discrete- or 24 toon-shaded image into a smooth 3D normal or height field that 25 matches the discrete shading and is consistent with viewer ex${ }_{26}$ pectations (Figure 2). As this is a severely underconstrained ${ }_{27}$ problem, our aim is to create plausible normal fields with min28 imal but necessary user input. Normal fields allow artists to 29 model shapes that do not correspond to physical 3D objects. ${ }_{30}$ Modeling in 3D is time intensive, and the drawn object may not

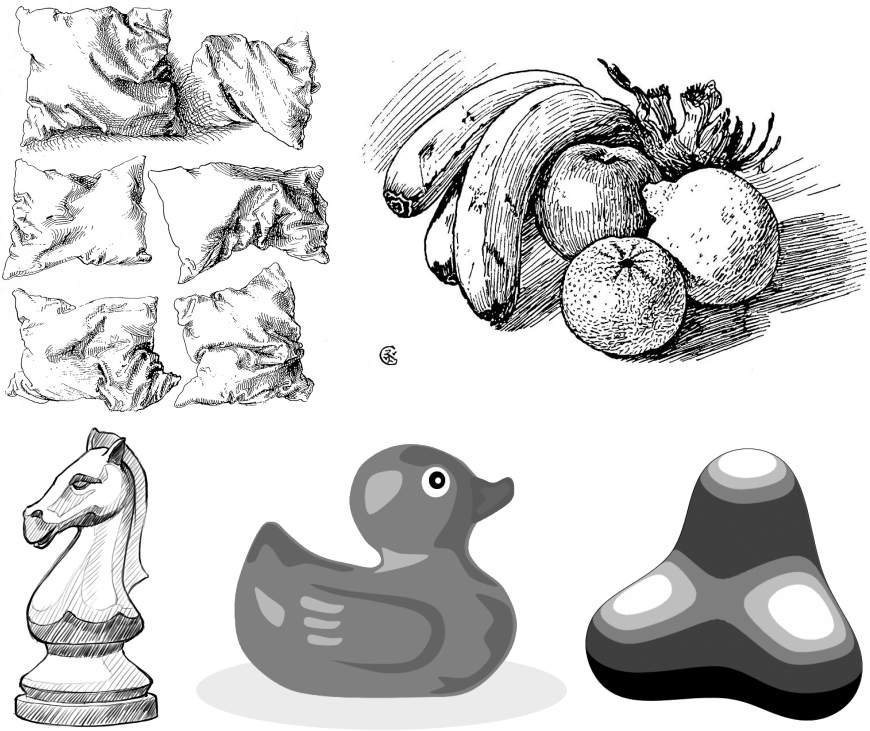

Figure 1: Motivational imagery includes drawings with discrete hatching, cartoon shaded and posterized art.

31 yet be realizable in 3D. In the early stage of a design process, 32 whole-object 3D consistency can be a distraction. Extending our ${ }_{33}$ recent work [6], we aim to lift a normal field into a height field ${ }_{34}$ given a cast shadow. We thus take as input the $2 \mathrm{D}$ silhouette of ${ }_{35}$ a smooth object along with one or more toon-shadings of the ${ }_{36}$ interior, each illuminated by a different directional light source ${ }_{37}$ (Figure 2a), and an optional cast shadow. Each toon shading is ${ }_{38}$ interpreted as a set of isophote curves (the iso-luminant boundз9 ary curves between toon-shaded bins) sharing the same light 


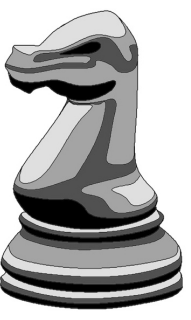

(a) Toon shaded input

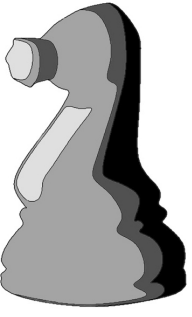

(b) 3D normals along isophotes

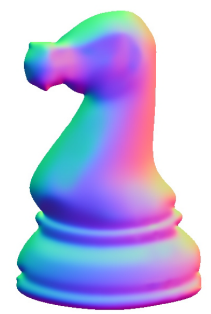

(c) 3D normal field

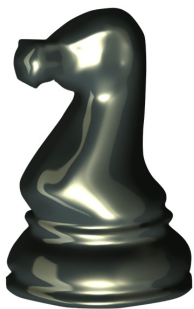

(d) 3D presentation renderings

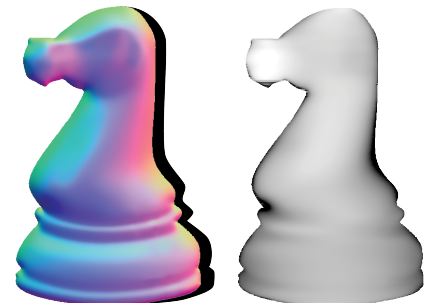

(e) Normal field with shadow (f) Height field

Figure 2: Quick line drawings with hatching or vector art frequently use a few discrete levels of tone or shading to convey smooth 3D shape (a). Artists use our tool to trace isophotes that separate discrete levels; our algorithm estimates smooth surface normals across the entire drawn objects. The normals along isophotes are interpolated to exhibit minimal variation and match viewer expectation (b). The resulting 3D normal fields (c) allow users to create 3D presentation renderings of objects using a variety of shading styles and lighting (d). With the addition of a cast shadow (e), we lift the normal field to obtain a $2.5 \mathrm{D}$ height field (f).

40 direction (Figure 2b). Artists pick light directions and draw ${ }_{41}$ isophote shapes, where the $2 \mathrm{D}$ isophote is indicative of its $3 \mathrm{D}$ 42 shape and the 3D surface normals change smoothly along the ${ }_{43}$ isophotes with minimal variation [4]. The boundary of the cast ${ }_{44}$ shadow is used to lift the normal field into a height field.

${ }_{45}$ Our 2D drawing interface (Section 4) provides artists with ${ }_{46}$ functionality for creating and editing silhouettes, isophotes, shad${ }_{47}$ ing values, and light directions. Light directions that best fit the ${ }_{48}$ shading values and 3D normals at silhouette/isophote intersec49 tion points can be automatically computed. 3D rendered results ${ }_{50}$ based on the surface normals computed by our inverse toon ${ }_{51}$ shading algorithm can be evaluated by the artist and undesirable 52 normals interactively corrected.

53 Our algorithm (Section 5) begins by computing well-defined ${ }_{54} 3 \mathrm{D}$ normals along the silhouette and at intersections between ${ }_{55}$ isophotes from different lights. (Isophotes from the same light ${ }_{56}$ direction do not typically intersect.) We then smoothly propagate ${ }_{57}$ these $3 \mathrm{D}$ normals along the $2 \mathrm{D}$ isophotes, and further refine the 58 normals along iteratively estimated 3D isophotes. Finally, we ${ }_{59}$ diffuse the normals throughout the interior of the shape. If a 60 cast shadow and accompanying light direction are provided, we ${ }_{61}$ integrate the normal field with the knowledge that the shadow 62 boundary is the object's silhouette from the light direction.

63 We perform two perceptual studies (Section 7) to measure the ${ }_{64}$ following: (I) consistency and accuracy of viewers' perceived 65 surface normals along isophotes taken from ground truth $3 \mathrm{D}$ ${ }_{66}$ data, and (II) artists' ability to draw isophotes. Our algorithm ${ }_{67}$ produces results comparable to viewers of the above study, vali${ }_{68}$ dating our approach. We evaluate our interface with user testers 69 and by creating a set of plausible 3D renderings created using 70 our interface (Section 8). We conclude with a summary of our 71 technique's limitations (Section 9).

72 Our chief contribution is formulating the novel problem of 73 inverse toon shading, to which we present a plausible, interactive 74 modeling solution: specifically, we develop an interactive tool 75 for drawing and shading silhouettes and isophotes, that guides 76 artists towards geometrically valid input; an algorithm that com77 putes a smooth 3D normal field to match the toon shaded input; 78 an algorithm that lifts a normal field with a shadow into a height 79 field; and perceptual studies that measures humans' ability to per80 ceive smooth surface normals along isophotes and draw accurate 81 isophotes.

\section{${ }_{82}$ 2. Related Work}

${ }_{83}$ Presentation rendering of sketches. Concept or gesture sketches 84 are quick line drawings with discrete shading cues, widely used 85 to rapidly explore ideas, that are then refined and shaded for ${ }_{86}$ presentation to the client $[4,3]$. While a finalized concept will ${ }_{87}$ indeed be turned into a 3D model for further processing, working ${ }_{88}$ directly with sketches facilitates rapid design iteration. Design89 ers commonly use painting tools such as Adobe Photoshop to 9o shade their sketches. Fast colorization and shading tools propa${ }_{91}$ gate scribbles [7] or diffuse color and shading using a few vector 92 primitives $[8,9]$. Despite these advances, painting convincing ${ }_{93}$ 3D-like shading requires extraordinary skill and time. Instead, 94 like Lumo [10], CrossShade [5], we go a step further: computing ${ }_{95}$ a 3D normal field from a 2D silhouette and a few other curves 96 and constraints. Lumo and its extensions [11, 12], based on ${ }_{97}$ silhouettes and internal contours, are well suited to amorphous 98 organic shapes. CrossShade, in contrast, is based on shape cross99 sections, ideal for structured man-made objects. Our work is 100 complementary to these systems in spirit but based on isophotes 101 and shading, which can be used to depict a mix of organic and 102 man-made forms (Figures 2, 14, 17). Several interfaces for spec${ }_{103}$ ifying normal fields $[13,14,15]$ have also been proposed. In our 104 tool, users specify the brightness of each isophote, which is a 105 single value rather than a smoothly changing 3D direction. Like ${ }_{106}$ CrossShade, however, our method builds upon the traditional 107 sketching workflow by using discrete tone shading commonly 108 employed by artists.

109 Shape from Shading and Sketching 3D models. A well-studied 110 and challenging Computer Vision problem concerns the recov111 ery of a height field from a 2D image of an object (see [16] for 112 a survey). Shape from shading approaches make this problem 113 tractable by assuming that the image contains only luminance 114 information from a single light source, and that the object is Lam115 bertian (perfectly diffuse). The related problem, when images 116 from multiple illumination directions are provided, is called Pho117 tometric Stereo [17]. While our problem input also comprises 118 shading information from one or more light directions, shape 119 from shading approaches are largely inapplicable as our input is 120 very sparse (luminance along a few isophotes) and contains in${ }_{121}$ evitable sketching inaccuracy. The goal of 3D rendering a sketch 122 is also disparate from height field or 3D model reconstruction. 
${ }_{123}$ In the former, the 2D sketched curves are preserved and any ${ }_{124}$ geometric inaccuracies are absorbed into the 3D normal field, 125 whereas in the latter the 3D output must typically deviate from ${ }_{126}$ the $2 \mathrm{D}$ sketch to be geometrically precise [18]. Our problem ${ }_{127}$ shares the general goal of inflating a flat 2D curve with sketch-, 128 image-, projection-, or silhouette-based approaches to creating ${ }_{129}$ 3D models [13, 19, 20, 21, 22, 23, 24, 25]. 3D sketch modeling ${ }_{130}$ research has also noted the use of shading as a handle for editing ${ }_{131}$ a 3D shape by editing its smooth shading [26]; their approach ${ }_{132}$ requires a pre-existing $3 \mathrm{D}$ shape and is not designed to create 133 one purely from shading.

${ }_{134}$ Isophotes and Reflection Lines. Fair surface design often em${ }_{135}$ ploys isophotes and reflection lines [27] to directly visualize ${ }_{136}$ the continuity of the surface gradient [28]. Deslandes and Bon${ }_{137}$ ner [29] describe an approach for exactly specifying reflection ${ }_{138}$ lines by modifying an existing 3D shape. Loos et al. [30] create a ${ }_{139}$ surface (height field) from desired illumination gradients defined 140 everywhere on the surface (equivalent to specifying isophote ${ }_{141}$ spacing). Tosun et al. [31] present a real-time solution to a 142 variant of this problem, in which an initial surface is modified. ${ }_{143}$ These illumination-gradient approaches allow control over the ${ }_{144}$ spacing and direction of isophotes, whereas our setting takes ${ }_{145}$ as input a sparse set of $2 \mathrm{D}$ projections of isophotes, without ${ }_{146}$ knowledge of the illumination gradient.

${ }_{147}$ Toon shading and NPR. There is a large body of work in non${ }_{148}$ photorealistic rendering [32] that addresses the inverse of our ${ }_{149}$ problem: producing discrete or toon shaded imagery in a variety 150 of artistic styles, even using isophote information [33], from a ${ }_{151} 3 \mathrm{D}$ scene or realistically shaded image.

\section{3. Definitions and Model Assumptions}

153 In toon shading or discrete tone shading, the surface illumina154 tion value is rounded to a discrete set of values. The boundary ${ }_{155}$ curves between bands of similar shading are isophotes. We pick 156 the isophote value to be the average tone of its two adjacent 157 bands. Assuming diffuse (Lambertian) illumination from a light 158 direction $l$, every isophote is a curve along which the surface 159 normal $n$ satisfies the equation $n \cdot l=k$, where $k$ is the constant ${ }_{160}$ tonal value. For a fixed $l$ and $k$, the set of satisfying normal vec${ }_{161}$ tors $n$ sweep out a cone around $l$, which we call the normal-cone ${ }_{162}$ (Figure 3). All 3D surface normals along the isophote lie on ${ }_{163}$ the normal-cone and can by parameterized by an angle value ${ }_{164} t \in[0,2 \pi)$ that sweeps a circle around the cone.

165 We use $x, y$ to denote the image plane with positive $z$ pointing 166 out of the plane. We also make several simplifying assumptions:

- The 3D shapes are smooth (at least tangent continuous).

- The light sources are directional and the shapes front-lit: $l_{z} \geq 0$.

- Shading is due to Lambertian reflection. In other words, there are no specular highlights. A specular hot-spot in 2D precisely defines a surface normal for a given view and light, and is easily incorporated into our approach.

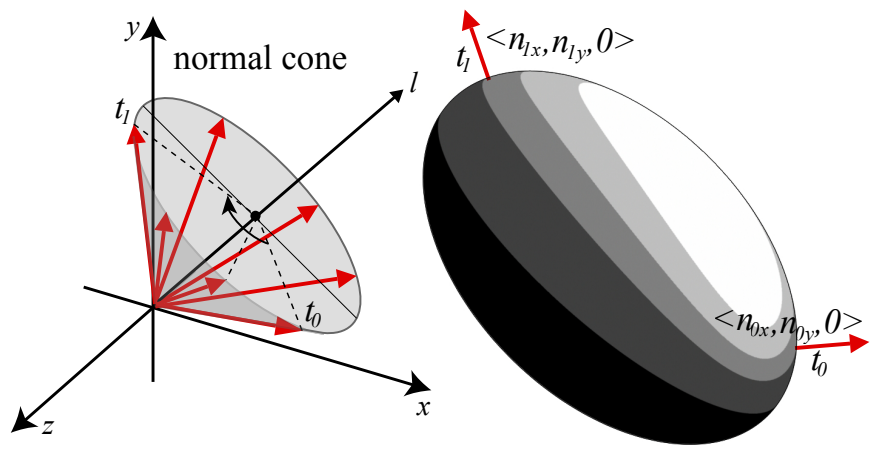

Figure 3: A normal cone is formed by iso-luminant surface normals at a constant angle to the light.

There are also no cast shadows present in the toon-shaded images; the shading only defines form shadows [2]. We ignore highlights and cast shadows to avoid the complexity of separating the Lambertian component from the overall surface illumination.

- There are no occluding contours. All surface normals in the interior of the silhouette have strictly positive $z$ components. Technically, surface normals on the occluding side of an internal contour are well-defined and normals on the occluded side can be handled by free-boundary diffusion. Internal contours, however, often cast form shadows locally on the shape, violating our previous assumption.

- Sketched 2D isophote shapes are descriptive of their 3D geometry: they are not drawn from a degenerate view; in $3 \mathrm{D}$, each isophote is as close to a planar curve as possible; and surface normals vary smoothly with fairness along the isophotes.

\section{4. Artist Workflow}

Our interface (see accompanying video) is essentially a 2D ${ }_{193}$ curve drawing and editing program. Artists begin by drawing 194 a 2D silhouette. They may then manually adjust a light source 195 using a light-sphere widget (top-right in Figure 4a-d) or simply 196 begin drawing isophotes (and an optional cast shadow).

Estimating the light direction: A single isophote intersecting the silhouette is sufficient to define the direction of the light. Surface normals at the isophote/silhouette intersections are well-defined 2D silhouette normals, $n_{0}=\left\langle n_{0_{x}}, n_{0_{y}}, 0\right\rangle$, $n_{1}=\left\langle n_{1_{x}}, n_{1_{y}}, 0\right\rangle$ (Figure 3, right). For distinct unit normals with the same illumination value $k$, the constraint $n_{0} \cdot l=n_{1} \cdot l=k$ implies that the $x y$ components of the light direction $l$ must be $\pm\left(n_{0}+n_{1}\right)$. In 3D, $l=\left\langle n_{0_{x}}+n_{1_{x}}, n_{0_{y}}+n_{1_{y}}, z\right\rangle$, normalized. For a given non-zero isophote value $k$, the constraint $n \cdot l=k$ results in a quadratic equation for $z$ :

$$
z= \pm \sqrt{\left(\frac{1+n_{0} \cdot n_{1}}{k}\right)^{2}-2\left(1+n_{0} \cdot n_{1}\right)}
$$

197 An average light direction can similarly be computed given 198 multiple isophotes that intersect the silhouette. For isophotes 


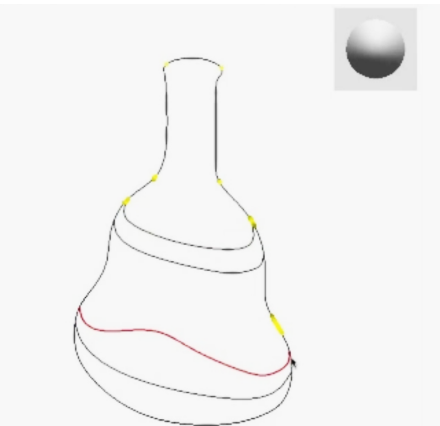

(a) isophote drawing

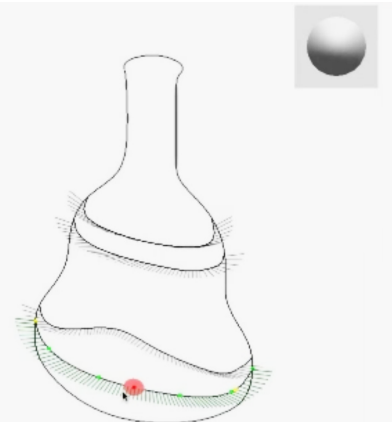

(b) 3D normal evaluation

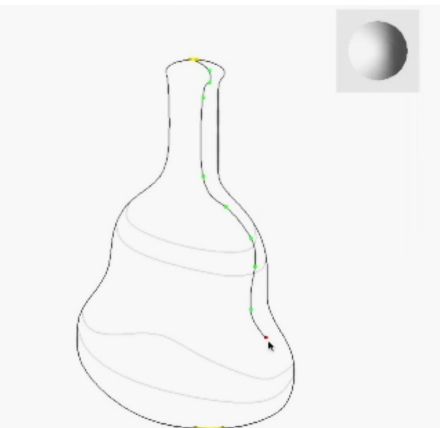

(c) isophotes from light \#2

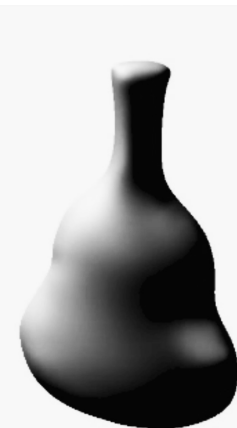

(d) 3D shading

Figure 4: Workflow snapshots: isophote drawing is guided by yellow regions where isophotes with a desired luminance value should intersect the silhouette (a); 3D normals along the isophote can be interactively viewed and corrected using the red gauge (b); isophotes from new light directions can be defined (c); and the normal field evaluated by shading $(d)$.

199 with illumination value $k=0, n_{0}=-n_{1}$ so only the $2 \mathrm{D}$ direction 200 of the light is fixed. At least one isophotes with non-zero $k$ value 201 is needed to define a 3D light direction.

202 Interaction: Once a light direction $l$ has been estimated or 203 manually defined and an isophote value $k$ specified, our inter204 face highlights points along the silhouette (in yellow) where the 205 desired isophote value is achieved $(n \cdot l=k)$ to guide the draw206 ing process towards consistent input (Figure 4a,c). Isophotes 207 are input as a sequence of points beginning and ending on the 208 silhouette; the points are interpolated with a natural cubic spline. 209 If the silhouette, light direction, or 210 isophote value is subsequently edited, 211 the isophote is affinely transformed on 212 the 2D drawing, so that the two old ${ }_{213}$ isophote-silhouette intersection points ${ }_{214}$ map to new isophote-silhouette intersec215 tions (as shown inset where the isophote

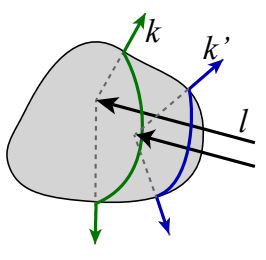
${ }_{216}$ value is changed from $k$ to $k^{\prime}$ ). The 3D normals along drawn 217 isophotes can be viewed interactively and corrected smoothly 218 at any point using a red gauge figure [34], constrained to ro${ }_{219}$ tate around the normal-cone (Figure 4b). Multiple toon images 220 drawn from different light directions further help constrain our ${ }_{221}$ problem (Figure 4c). Where isophotes from different light di222 rections intersect, the surface normal is one of two intersection ${ }_{223}$ directions of the two normal-cones (if they intersect). Intersect224 ing isophotes with non-intersecting normal-cones are visualized 225 as inconsistent. The 3D normal field from the current toon 226 shaded input can be computed at any time and evaluated using 227 3D shading under interactive lighting (Figure 4d). Additional 228 isophotes from the current 3D normal field can also be extracted 229 and subsequently edited. The cast shadow, for lifting normal 230 fields into height fields, is drawn as either a 2D boundary curve ${ }_{231}$ or as a $2 \mathrm{D}$ region whose boundary is automatically extracted.

\section{5. 3D Normal Field Algorithm}

233 Our algorithm proceeds in four stages as shown by the green ${ }_{234}$ path in Figure 5. The alternate paths suggest that intermediate 235 steps could be considered optional. However, we find empiri236 cally that this sequence of all four stages provides the best results

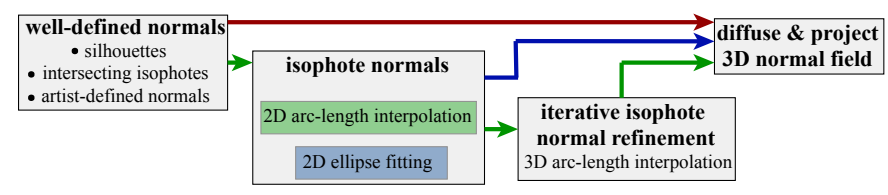

Figure 5: Stages of the 3D Normal Field Algorithm.

237 overall. Our proposed algorithm is thus: compute well-defined ${ }_{238}$ normals, interpolate normals along isophotes based on 2D arc239 length, iteratively re-interpolate normals based on 3D isophote 240 arc-length, and iteratively diffuse normals into the interior and ${ }_{241}$ project normals along isophotes back to the normal-cones.

\section{5.1. Well-Defined Normals}

243 The 3D surface normals along the silhouette of a smooth shape 244 as shown in (Figure 3, right) are $n=\left\langle n_{x}, n_{y}, 0\right\rangle$, where $\left\langle n_{x}, n_{y}\right\rangle$ 245 is the $2 \mathrm{D}$ silhouette normal. Where isophotes from different 246 light directions $l_{1}, l_{2}$ intersect, the surface normal $n$ is one of two 247 intersection directions of the two normal-cones obtained as the 248 solution to a quadratic equation from $n \cdot l_{1}=k_{1}, n \cdot l_{2}=k_{2}$, and $249\|n\|=1$. Assuming the isophotes are consistent and a solution 250 exists, our desired normal $n$ must have $n_{z}>0$. If only one of 251 the two normals has $n_{z}>0$ (both $n_{z}$ cannot be negative since 252 we assume front lighting), we select it as a normal constraint. ${ }_{253}$ If there are two normals with $n_{z}>0$, we select the normal 254 that is closer (the one with larger dot product) to the currently 255 estimated surface normal, and re-run the algorithm with the 256 normal constraint. If the normal cones do not intersect, we ask 257 the user for input adjustment.

\section{5.2. Estimating Normals Along Isophotes}

${ }_{259}$ With the aim of producing smoothly varying normals with 260 global fairness along isophote curves, we consider two alterna${ }_{261}$ tives. The first is a linear interpolation of the cone-angle along 262 an isophote between two known normals, which ensures min263 imally varying normals along the isophote. The second is a 264 reconstruction of isophotes in 3D as piecewise line segments 265 and circular arcs, producing fair isophotes of minimally varying 266 curvature. 
${ }_{267} 2 \mathrm{D}$ arc-length based cone-angle interpolation. As shown in Fig268 ure 3, surface normals along an isophote between two known nor269 mals $n_{0}$ and $n_{1}$, are minimally interpolated by linearly interpolat270 ing the cone angle $t$, between $t_{0}$ and $t_{1}$. We divide each isophote 271 into segments between normals computed in Section 5.1, and 272 perform piecewise linear interpolation. Interpolation of $t$ is cir273 cular and can take place around one of two directions between $t_{0}$ 274 and $t_{1}$. Note that while $n_{0 z}$ and $n_{1 z}$ for any two known normals 275 are non-negative, there may be a segment of $t$ values where the 276 cone-angle normals $n$ are back-facing $\left(n_{z}<0\right)$. If such a segment 277 exists, we pick the interpolation direction where the cone-angle 278 remains positive, or else we pick the direction with the smaller 279 interpolation angle. While this approach works well for simple 280 isophote shapes, it is unacceptable for complex isophotes as ${ }_{281}$ shown in Figure 6. As the 2D isophote is indicative of its 3D 282 shape, it is reasonable to expect that, relative to convex seg283 ments, the surface normal in flat and concave regions of the 2D 284 isophote, will stay constant and reverse interpolation direction, 285 respectively.

We thus further segment the 2D isophote segment into convex, flat and concave regions according to McCrae et al. [35] (Figure 6). Let there be $m$ such segments $S_{0}, \ldots, S_{m}$. Let the $2 \mathrm{D}$ turning angle between the $2 \mathrm{D}$ tangent at the start and end of the $i$-th segment be $\phi_{i}$. If $\Delta t$ is the overall change in cone-angle, we define the cone-angle change for the $i$-th segment $\delta t_{i}$ as

$$
\delta t_{i}=\frac{\phi_{i} \Delta t}{\sum_{0}^{m} \phi_{i}} .
$$

286 By definition of $\phi_{i}, \delta t_{i}$ will now be zero for flat segments and ${ }_{287}$ of opposite sign for convex and concave segments. Within each ${ }_{288}$ segment $S_{i}, \delta t_{i}$ is still linearly interpolated by $2 \mathrm{D}$ arc-length, as 289 the relationship between $2 \mathrm{D}$ and 3D curvature is not necessarily 290 proportional due to foreshortening.

There are still two issues with the above formulation that need to be resolved. First, the above formulation is unstable when the overall start and end tangent for the isophote segment are nearly equal $\sum_{0}^{m} \phi_{i} \approx 0$. Barring straight-line isophotes with constant surface normal, we rarely encounter this scenario in practice and handle it by asking the user to specify a normal within the segment, breaking it into two well-defined segments. Second, the cone-angle interpolation is no longer guaranteed to lie within $\Delta t$ (due to direction reversal in concave regions) and can result in backfacing normals. Let the isophote attain its most negative normal after the $i$-th segment $S_{i}$ at a $t$ value $t_{\min }$, i.e. $t_{\min }=t_{0}+\sum_{0}^{i} \delta t_{i}$. Let its adjacent $t$ value where the normal is in the viewplane $n_{z}=0$ be $t_{\text {sil }}$. We can compute new scaled interpolation angles for segment from $0 \ldots i$ to be

$$
\delta t_{i} \frac{t_{\mathrm{sil}}-t_{0}}{t_{\min }-t_{0}}
$$

and for segments from $i+1 \ldots m$ to be

$$
\delta t_{i} \frac{t_{1}-t_{\mathrm{sil}}}{t_{1}-t_{\mathrm{min}}} .
$$

291 It is straightforward to verify that the cone-angle now interpo292 lates from $t_{0}$ to $t_{\mathrm{sil}}$ at the end of segment $S_{i}$, and from $t_{\mathrm{sil}}$ to $t_{1}$ 293 and the end of segment $S_{m}$, with strictly front-facing normals.

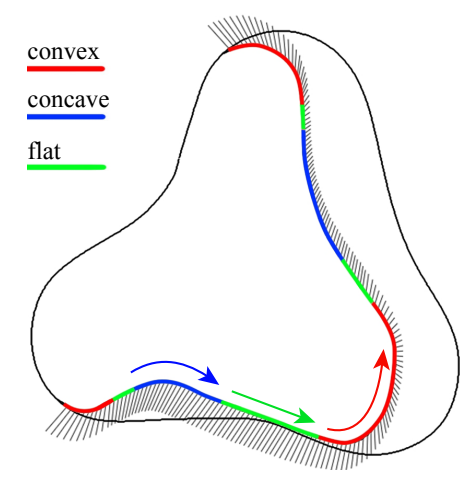

Figure 6: Cone-angle interpolation stops in flat green segments of an isophote and reverses direction in concave blue regions.

For closed interior isophotes, we require that they be inter295 sected by at least one isophote from a different light, so that it 296 may be segmented by at least two well-defined normals.

297 Elliptical arc fitting. We are motivated by the desire to fit sim${ }_{298}$ ple 3D surface primitives whose isophotes would match the ${ }_{299}$ drawn isophotes. Unfortunately even the isophotes of simple ${ }_{300}$ 3D ellipsoids are generally non-planar curves called spherical 301 cyclic curves. We thus pick a line and a circle, the simplest 3D 302 isophotes that we can reconstruct from their drawn 2D projec${ }_{303}$ tions. If we assume a $2 \mathrm{D}$ straight line is a straight line in $3 \mathrm{D}$ 304 [18], the isophote segment lies on a ruled surface with constant 305 surface normal.

Let us model a circular arc in object space as $(0, \cos (a), \sin (a))$ for some range $\left[a_{0}, a_{1}\right]$. (In object space, the circle lies on a plane perpendicular to the $X$ axis.) The surface normal for a ribbon around this arc is

$$
\frac{\langle x, \cos (a), \sin (a)\rangle}{\sqrt{1+x^{2}}}
$$

for some $x$. The above circular arc becomes an ellipse in the $x y$ image plane as a result of rotation $R_{y}(u)$ by angle $u$ about $Y$ and $R_{z}(v)$ by $v$ about $Z$. The aspect ratio of this ellipse is now $\sin (u)$, and the angle of its minor axis to the $X$-axis is $v$. Given any 2D elliptic arc, we can determine its transformation $M=$ $\left(R_{z}(v) R_{y}(u)\right)^{-1}$ from image to the object space of the circular arc from $u$ and $v$. We can thus transform the light $l$ from image to object space $l^{\prime}=M l$. In object space the isophote equation is

$$
x l_{x}^{\prime}+\cos (a) l_{y}^{\prime}+\sin (a) l_{z}^{\prime}=k \sqrt{1+x^{2}}
$$

306 where $k$ is the isophote value, and $x$ can be solved as a quadratic 307 equation whose roots are functions of the circular-arc parameter ${ }_{308} a$. If there are no solutions, we snap to the solution for the ${ }_{309}$ closest $k$ value, and project this solution normal back to the ${ }_{310}$ desired normal-cone. As a sanity check, when the light direction ${ }_{311}$ is perpendicular to the circular arc, $x$ is a constant function of ${ }_{312} k$ and the ribbon around the circular isophote is locally cone 313 shaped.

314 For each convex and concave isophote segment, we fit a min315 imal number of 2D ellipses given an error tolerance ( 3 pixels 316 in our implementation) [36]. As pointed out by the recent ap${ }_{317}$ proach of Company et al. [37], the approach we use is slower 
318 than needed for some data, not as robust as needed for other ${ }_{319}$ data, and our error tolerance is not perceptually motivated. As 320 an alternative, we could use the approach of Company et al. [37], 321 which chooses between three different ellipse fitting approaches 322 based on perceptual accuracy and running time and returns a ${ }_{323}$ perceptual goodness-of-fit. For each ellipse, which we assume 324 to be the projection of a circle, there are 4 possible circle plane 325 normals ( 2 tilt directions for angle $v$, and convex or concave 326 normals). Segments that intersect the silhouette are convex by 327 virtue of a smooth 3D shape and have two choices. Additional 328 criteria are: the normal at the shared point between adjacent seg329 ments should match; the normals within each segment should ${ }_{330}$ vary smoothly; the normals have positive $z$ components. We ${ }_{331}$ define a fitness metric for any combination to be the overall sum ${ }_{332}$ of absolute differences between cone-angle $t$ at the common ${ }_{33}$ point of adjacent segments, sum of the standard deviation of ${ }_{334}$ cone-angle $t$ within each segment, and the percent of normals 335 with negative $\mathrm{z}$ components. We permute all $2 \cdot 2 \cdot 4^{\# \text { segments }-2}$ ${ }_{336}$ possible combinations and pick the fittest.

\section{${ }_{337}$ 5.3. Iterative $3 D$ arc-length based interpolation}

The 2D arc-length based interpolation of Section 5.2 ignores foreshortening effects. As a result, normals along the 3D circular isophote of a sphere, would turn quickly near the silhouette and appear flat in the interior, as dictated by the arc length of a 2D ellipse. Alternatively, the ellipse fitting algorithm has discontinuities at segment junctions. We improve these estimated normals by iteratively estimating a 3D isophote. An estimated surface normal $n$ along an isophote is perpendicular to its 3D isophote tangent $\left\langle t_{x}, t_{y}, t_{z}\right\rangle$, as

$$
t_{z}=-\frac{n_{x} t_{x}+n_{y} t_{y}}{n_{z}}
$$

where $\left\langle t_{x}, t_{y}\right\rangle$ is the known 2D isophote tangent. Given surface normals along an isophote, we compute 3D tangents as just described. Near silhouettes and where $n_{z}<\epsilon$ (we use $\epsilon=0.004$ ), we compute $t_{z}$ using l'Hospital's formula and finite differences, i.e.

$$
t_{z}=-\frac{\Delta\left(n_{x} t_{x}+n_{y} t_{y}\right)}{\Delta n_{z}} .
$$

3з8 We then smooth $t_{z}$ minimally by neighbor averaging (strength ${ }_{339} 0.3$ ), recompute normals using the equations $n \cdot t=0, n \cdot l=k$, $340\|n\|=1$, and repeat the process for a fixed number of iterations ${ }_{341}$ or until the normals converge.

\section{5.4. Diffuse and Project across the Shape}

343 We treat the interior of the shape as a 3D normal field ${ }_{344} \mathbb{R}^{2} \rightarrow \mathbb{R}^{3}$. The known $\left\langle n_{x}, n_{y}, n_{z}\right\rangle$ values for normals on the 345 silhouette and at isophote intersections are boundary constraints 346 for a diffusion process. Estimated normals along isophotes, and 347 user provided additional normals, are included as soft constraints. ${ }_{348}$ After diffusion, the computed normals along isophotes may have ${ }_{349}$ deviated from the normal cone. We project them onto the normal 350 cone and update the soft constraints to the new normals. We ${ }_{351}$ repeat these diffuse and project steps until convergence. Unlike
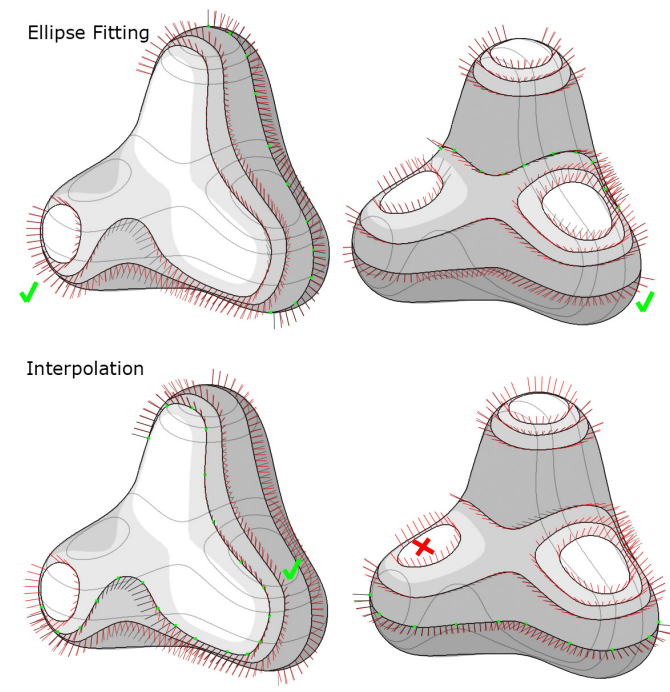

Figure 7: Ellipse fitting and 2D arc-length based cone-angle interpolation produce complementary results.

352 our previous steps, the diffuse-and-project method does not rely 353 on specific shape properties of isophotes.

We presented two approaches in Section 5.2 for initial surface 355 normal estimation along isophotes. While we generally promote ${ }_{356} 2 \mathrm{D}$ arc-length based interpolation for its robust simplicity, el357 lipse fitting has the advantage that it does not require any known 358 normals along the isophote. It is thus applicable to closed and 359 isolated isophotes for which there are no normals to be interpo${ }_{360}$ lated. It also complements $2 \mathrm{D}$ arc-length based interpolation by ${ }_{361}$ implicitly handling view foreshortening. Figure 7 marks regions 362 where one approach tends to perform better than the other.

363 As Figure 5 suggests, one could skip normal estimation along 364 isophotes (Sections 5.2 and 5.3) altogether and simply diffuse 365 well-defined normals throughout the shape, projecting the dif366 fused isophote normals onto their normal-cone. Theoretically, ${ }_{367}$ we expect poorer results as this process will result in smooth 368 normals along isophotes, but ones that might lack fairness, os369 cillating back and forth arbitrarily around the normal-cone. We 370 do indeed observe this behaviour in practice. This is discussed 371 in Section 7 and visualized in Figure 9. Both isophote normal 372 estimation stages (Sections 5.2 and 5.3) have a positive impact 373 on the 3D normal field construction.

\section{${ }_{374}$ 6. Height Field Algorithm}

375 Our algorithm for producing a height field $H(x, y)$ takes as 376 input a normal field $N(x, y)$, a light direction $l$, and a hard cast 377 shadow $\mathbf{S}$. Form shadows, in which the shadow is cast onto the 378 shape itself, are left as future work. We assume that the surface 379 is $C^{1}$ continuous and closed. We additionally assume that the ${ }_{380}$ shadow is cast onto the $z=0$ plane, although we relax this 381 constraint somewhat in a later stage.

Our algorithm begins by rotating the input around the $z$ axis such that the light direction lies in the $x z$ plane $\left(l_{y}=0\right)$ with $l_{x}<0$. (If the light is directly overhead, $l_{x}=l_{y}=0$, and there 


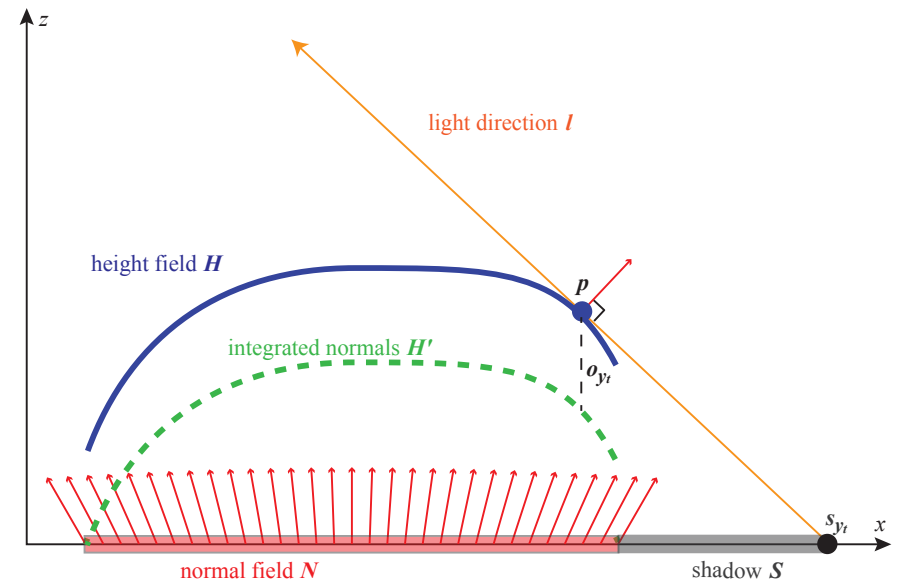

Figure 8: An illustration of the height field algorithm. An $x z$-plane slice of the normal field $N$ is integrated to obtain a relative height field $H^{\prime}$. The absolute height field $H$ is obtained by offsetting $H^{\prime}$ to graze the light direction $l$.

would be no cast shadow). Our algorithm processes each horizontal slice (row of pixels) $H\left(x, y_{t}\right)$ for each $y_{t}$, independently (Figure 8). For each slice, we collect the shadow and surface extents (1D connected components). For each surface extent, we find the boundary of the corresponding shadow extent $\mathbf{S}_{y_{t}, i}$ in the $+x$ direction:

$$
s_{y_{t}}=\max _{x} \text { such that } x \in \mathbf{S}_{y_{t}, i}
$$

382 (If the correct $s_{y_{t}}$ is occluded and the visible shadow boundary 383 is used instead, our algorithm will produce erroneous results 384 (Section 8).) The light that reaches $s_{y_{t}}$ grazes the surface at 385 some point $p=\left(x, y_{t}\right)$. Because the surface is $C^{1}$ continuous, ${ }_{386} l \cdot N(p)=0$. These two criteria inform our algorithm. By 387 integrating the normals along the slice, we obtain a $1 \mathrm{D}$ relative 388 height field $H^{\prime}$. (We integrate by treating pixels as piecewise 389 planar patches with the given normals, and cumulatively sum the $390 z$ values of the patch gradients.) We find the absolute height field 391 slice $H\left(x, y_{t}\right)=H^{\prime}\left(x, y_{t}\right)+o_{y_{t}}$ in two stages. Let $z(x)=m x+b$ 392 be the equation for the line parallel to the light direction and 393 passing through $s_{y_{t}}$. First, we find $x^{\prime}$ such that the offset $o_{y_{t}}^{\prime}=$ $394 z\left(x^{\prime}\right)-H^{\prime}\left(x_{0}, y_{t}\right)$ is minimized. This is the offset such that the 395 light grazes $H^{\prime}\left(x, y_{t}\right)+o_{y_{t}}^{\prime}$. Second, we incorporate the normal 396 condition. We find the $x^{\prime \prime}$ in the range $x^{\prime} \pm 10$ whose normal $397 N\left(x, y_{t}\right)$ angle with the light direction is most perpendicular $398\left(\right.$ closest to $\left.90^{\circ}\right)$. The final offset $o_{y_{t}}$ for extent is determined from 399 the offset at $x^{\prime \prime}: o_{y_{t}}=z\left(x^{\prime \prime}\right)-H^{\prime}\left(x^{\prime \prime}, y_{t}\right)$.

Denoising. To relax the assumption that the shadow is cast onto the $z=0$ plane, or to correct inaccuracies in user-drawn shadows, we optionally offset adjacent slices in $z$ to minimize the deviation

$$
\min _{c} \int_{a}^{b}\left|H\left(x, y_{t}\right)-H\left(x, y_{t}+1\right)+c\right|^{p} d x .
$$

400 For the $\ell^{2}$ norm $(p=2)$, the minimizer $c$ is the average dif401 ference $H\left(x, y_{t}\right)-H\left(x, y_{t}+1\right)$. For the $\ell^{1}$ norm $(p=1)$, the 402 minimizer $c$ is the median difference $H\left(x, y_{t}\right)-H\left(x, y_{t}+1\right)$. We 403 use the $\ell^{1}$ norm, as it is more robust to outliers.

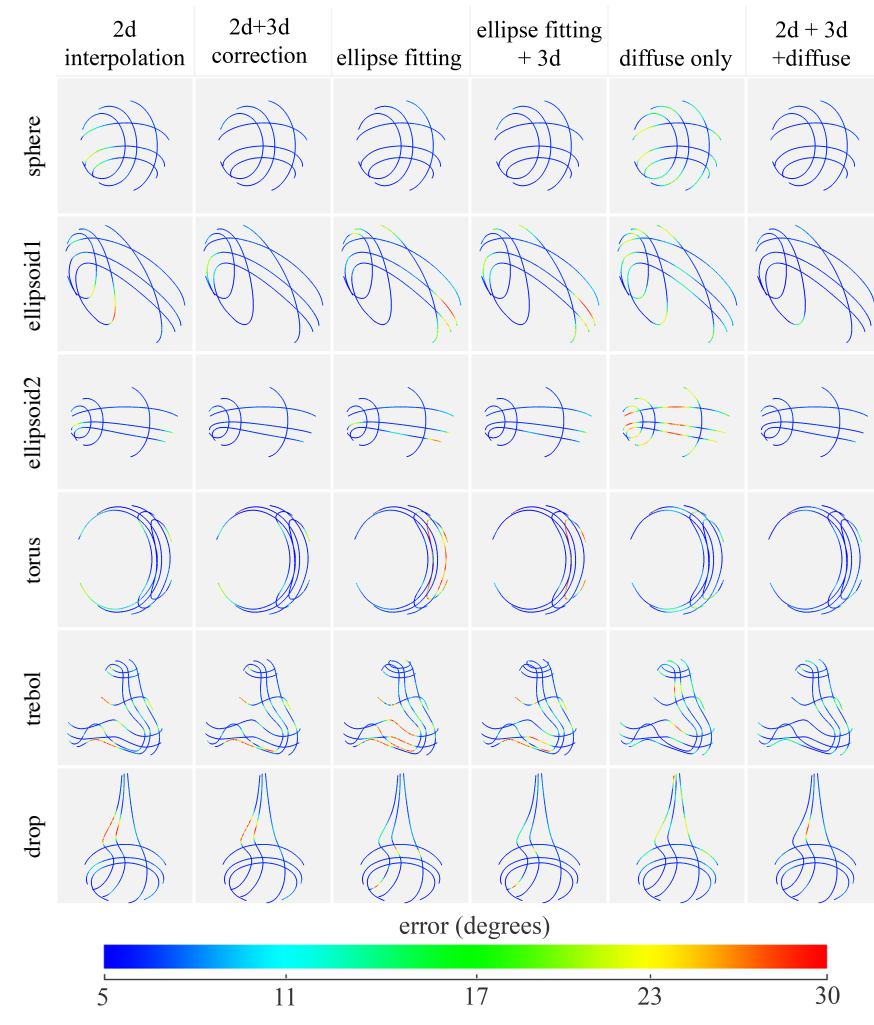

Figure 9: Impact of algorithm stages on shapes in Figure 13. We measure angular error along isophotes with respect to ground truth normals. A numerical summary is shown in Table 1.

\section{7. Validation}

405 Algorithm Evaluation. Figure 9 shows the impact of each stage 406 of the algorithm in Section 5 on the reconstruction error of 407 ground truth 3D data. Ground truth data in all of our experi408 ments was obtained from Autodesk Maya. Along each isophote, 409 we measured the angular error with respect to ground truth (Ta410 ble 1). We evaluated both isophote normal estimation algorithms 411 (Section 5.2) by themselves, and combined with our iterative ${ }_{412}$ refinement stage (Section 5.3). In isolation, 2D arc-length based ${ }_{413}$ interpolation performed comparably to ellipse fitting. Iterative ${ }_{414} 3 \mathrm{D}$ refinement decreased the median error for both approaches, 415 but more significantly for 2D interpolation (correcting foreshort416 ening errors). Our diffuse-and-project algorithm for propagating 417 normals through the shape produces poor results in isolation, 418 typically worse than our isophote normal estimation algorithms ${ }_{419}$ without 3D refinement. Combining the stages of $2 \mathrm{D}$ arc-length 420 interpolations, 3D refinement and diffuse-and-project produced ${ }_{421}$ the lowest median error of $\approx 5.5^{\circ}$.

Figure 10 shows our algorithm to be robust to the incremental 423 addition and removal of isophotes, with predictable changes in 424 the resulting 3D normal field.

425 Perceptual Study I. We carried out the first perceptual study to 426 answer the following three questions about toon shaded images:

${ }_{427}$ Q1 Do humans perceive isophote normals consistently?

${ }_{428} \mathbf{Q 2}$ Are humans accurate (consistent with ground truth)? 


\begin{tabular}{|l|l|l|c|c|c|c|c|c|c|c|c|c|}
\hline & \multicolumn{2}{|c|}{$2 \mathrm{~d}$} & \multicolumn{2}{c|}{$2 \mathrm{~d}+3 \mathrm{~d}$} & \multicolumn{2}{c|}{ ellipse fitting } & \multicolumn{2}{c|}{ ellipse + 3d } & \multicolumn{2}{c|}{ diffuse only } & \multicolumn{2}{c|}{ 2d + 3d + diffuse } \\
\cline { 2 - 13 } & med. & std. & med. & std. & med. & std. & med. & std. & med. & std. & med. & std. \\
\hline sphere & 6.51 & 4.55 & 4.94 & 3.35 & 4.99 & 3.4 & 4.94 & 3.35 & 8.85 & 5.44 & 4.9 & 3.24 \\
ellipsoid1 & 6.33 & 5.41 & 5.76 & 4.11 & 6.71 & 6.34 & 6.6 & 6.35 & 8.52 & 5.5 & 5.3 & 3.45 \\
ellipsoid2 & 6.29 & 4.05 & 5.51 & 3.38 & 6.28 & 5.19 & 5.84 & 3.81 & 14.11 & 14.52 & 5.46 & 3.3 \\
torus & 7 & 5.3 & 6.06 & 5.09 & 7.9 & 12.97 & 6.27 & 12.31 & 7.6 & 5.05 & 5.97 & 4.89 \\
trebol & 7.21 & 9.21 & 7.04 & 9.57 & 8.15 & 11.63 & 7.39 & 9.69 & 7.7 & 5.73 & 6.4 & 4.63 \\
drop & 7.09 & 7.48 & 6.1 & 6.95 & 6.27 & 4.92 & 5.93 & 4.56 & 7.27 & 5.7 & 5.86 & 5.1 \\
\hline
\end{tabular}

Table 1: Summary of our experiments comparing each step of our surface normal estimation algorithm to ground truth. The median (med.) and standard deviation (std.) are shown. A visual summary can be found in Figure 9.

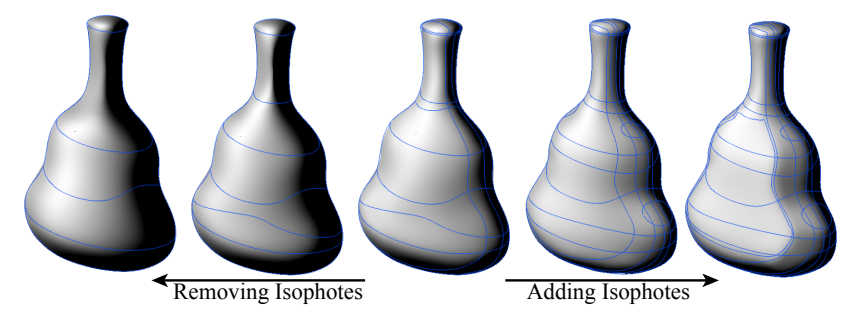

Figure 10: Impact of isophote addition and removal on the 3D shaded result

${ }_{429}$ Q3 Is our algorithm's accuracy comparable to humans?

${ }_{430}$ Our subject pool consisted of 11 artists and 9 non-artists/non${ }_{431}$ designers, all with some CG background. Participants were ${ }_{432}$ shown a description of toon shading and then completed a series 433 of 52 gauge figure orientation tasks [34] over toon shaded objects. ${ }_{434}$ Gauge figures were always placed on the isophote between 435 two discrete tones. The first 26 gauge figures were shown in ${ }_{436}$ isolation with only two tonal bands adjacent to the isophote. ${ }_{437}$ The entire object was shown for the last 26 gauge figures. The ${ }_{438}$ first and second half of each group of 26 gauge figures were 439 either (randomly) freely orientable or constrained to lie on the 440 normal-cone. The constrained scenario, giving participants one ${ }_{441}$ degree of freedom, corresponds to our algorithm's constrained ${ }_{442}$ solution space. The unconstrained scenario allows us to compare ${ }_{443}$ our study's results to previous gauge figure experiments. Table 2 444 summarizes our results. Our automatic approach for estimating 445 normals along isophotes substantially outperformed humans 446 (Table 1 and 2).

${ }_{447}$ Findings:

- Human perception of isophote normals are more consistent with each other than accurate to ground truth. Humans have a tendency to orient the surface normal perpendicular to the isophote curve in 2D. In many cases the spread of gauges ${ }^{469}$ is not centered around the ground truth normal, but biased 470

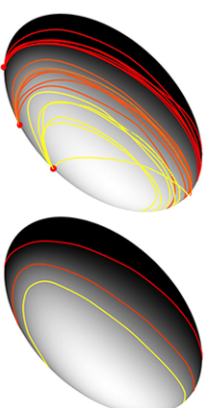

(a)

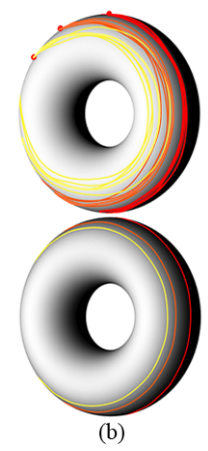

(b)

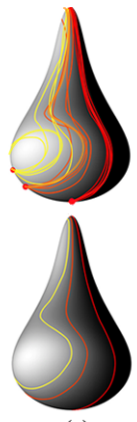

(c)

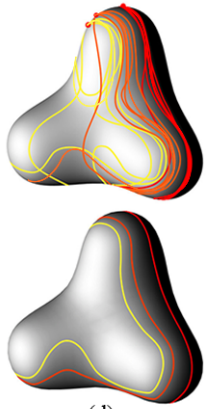

(d)
Figure 12: Second perceptual study results. Top row: artist-drawn isophotes. Bottom row: ground truth isophotes. Isophotes of value $k=0,0.5$, and 0.7 are shown in red, orange, and yellow, respectively.

towards the 2D curve's normal direction (Figure 11a-c).

- Human accuracy at the crest shadow (tone value $k=0$ ) is notably higher than along isophotes with lighter values. The average median error of all $\mathrm{k}=0$ test cases is $14^{\circ}$, and $20.3^{\circ}$ for other values. For non-extreme light directions, the crest shadow's shape is closest to the silhouette, where the $2 \mathrm{D}$ shape is indeed representative of the 3D normal. Human consistency with our algorithmic result is higher than with ground truth. While perhaps surprising, we designed our algorithm around the assumption that 2D isophote shape is indicative of local 3D geometry, which is inspired by human perception [4].

- Isophotes are ambiguous when the complete shape is not shown (Figure 11d,e).

- Human consistency and accuracy is better when the gauge is constrained to lie on the normal-cone. The artist pool reported $\approx 2^{\circ}$ better consistency and accuracy than nonartists.

${ }_{471}$ Perceptual Study II. We carried out a second study to assess 472 artists' ability to draw isophotes consistently and accurately. Our 473 subject pool consisted of 5 artists. Participants were shown 4 ${ }_{474}$ Lambertian shaded shapes and then asked to draw 3 isophotes $475(k=0,0.5$, and 0.7$)$ for each shape. The starting point of each 476 isophote was marked as a red dot on the silhouette. The results 477 of this study are summarized in Figure 12.

Figure 11: First perceptual study results. Red: user placed normals. Green: ground truth normals.

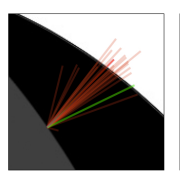

(c)

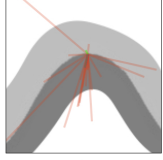

(d)

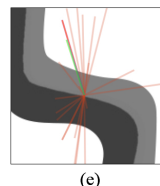

$478 \quad$ Findings: 


\begin{tabular}{|l|c|c|c|c|c|c|}
\hline & $\begin{array}{c}\text { Pers. } \\
\text { intra } \\
\text { user }\end{array}$ & $\begin{array}{c}\text { Cons. } \\
\text { inter } \\
\text { user }\end{array}$ & $\begin{array}{c}\text { Acc. } \\
\text { user/GT }\end{array}$ & $\begin{array}{c}\text { Acc. } \\
\text { user/GT } \\
\text { outliers }\end{array}$ & $\begin{array}{c}\text { Cons. } \\
\text { user/algo }\end{array}$ & $\begin{array}{c}\text { Acc. } \\
\text { algo/GT }\end{array}$ \\
\hline Complete (constrained movement) \\
\hline median & 7.1 & 8.2 & 16.4 & 15 & 14.5 & 5.6 \\
mean & 10.1 & 14.1 & 20.2 & 17.9 & 15.6 & 5.8 \\
std. dev. & 16.3 & 17.1 & 16.8 & 13.5 & 13.4 & 4.2 \\
samples & 98 & 1616 & 314 & 299 & 314 & 65 \\
\hline Complete (free movement) \\
\hline median & 9.3 & 16 & 19.5 & 18.8 & 19.1 & \\
mean & 10.5 & 19.7 & 22.3 & 20.1 & 21.2 & \\
std. dev. & 15.3 & 16.1 & 16.2 & 12.9 & 16.1 & \\
samples & 186 & 6260 & 628 & 597 & 628 & \\
\hline Partial (constrained movement) & & \\
\hline median & 7.2 & 8.2 & 17.3 & 16 & 15.9 & \\
mean & 11.4 & 15.4 & 23.5 & 20.5 & 20.2 & \\
std. dev. & 17.5 & 21.5 & 20.7 & 15.8 & 19.8 & \\
samples & 103 & 1681 & 333 & 317 & 333 & \\
\hline Partial (free movement) & \multicolumn{7}{|l|}{} \\
\hline median & 7.6 & 18.3 & 23.5 & 22.3 & 21.2 & \\
mean & 11.9 & 24.1 & 27.1 & 24.4 & 24.3 & \\
std. dev. & 18.3 & 20.8 & 19.6 & 15.6 & 15.1 & \\
samples & 205 & 6580 & 665 & 632 & 665 & \\
\hline
\end{tabular}

Table 2: Summary of our validation data. (Left to right) persistence same user, consistency between users, accuracy with respect to ground truth (GT), accuracy with worst $5 \%$ of errors removed, consistency with respect to our solution, accuracy of our solution with respect to ground truth.

\section{8. Results}

498 Implementation Details. Our prototype was implemented on a 499 Intel Core i7 $2.3 \mathrm{GHz}$ processor with $8 \mathrm{~GB}$ RAM. The inter500 face canvas resolution is 700 by 700 pixels. Typical time for 501 normal estimation by 2D interpolation is negligible. Ellipse 502 fitting takes on the order of 6 seconds. 3D refinement takes 1 ${ }_{503} \mathrm{sec}$ (75 iterations) and diffusion takes approximately 2 minutes 504 (5 iterations).
- Artists have a good understanding of isophote properties when presented with toon-shaded examples. All user-drawn isophotes from a single light source are continuous and nonintersecting.

- Artist-drawn isophotes are highly consistent at the crest shadow $(k=0)$ and less consistent at other $k$ values. This observation matches the result from Perceptual Study I. For more complicated shapes (Figure 12d), artist consistency is notably lower.

- Artist-drawn isophotes are distributed closely to the ground truth. However, most drawn isophotes match a $k$ value higher than the assigned value. This is in line with Perceptual Study I, which found that humans overestimate $k$.

- When an isophote approaches the silhouette, its tangent starts to follow the tangent of the silhouette. The more perpendicular the light direction is to the image plane, the longer the segment of isophote drawn this way.

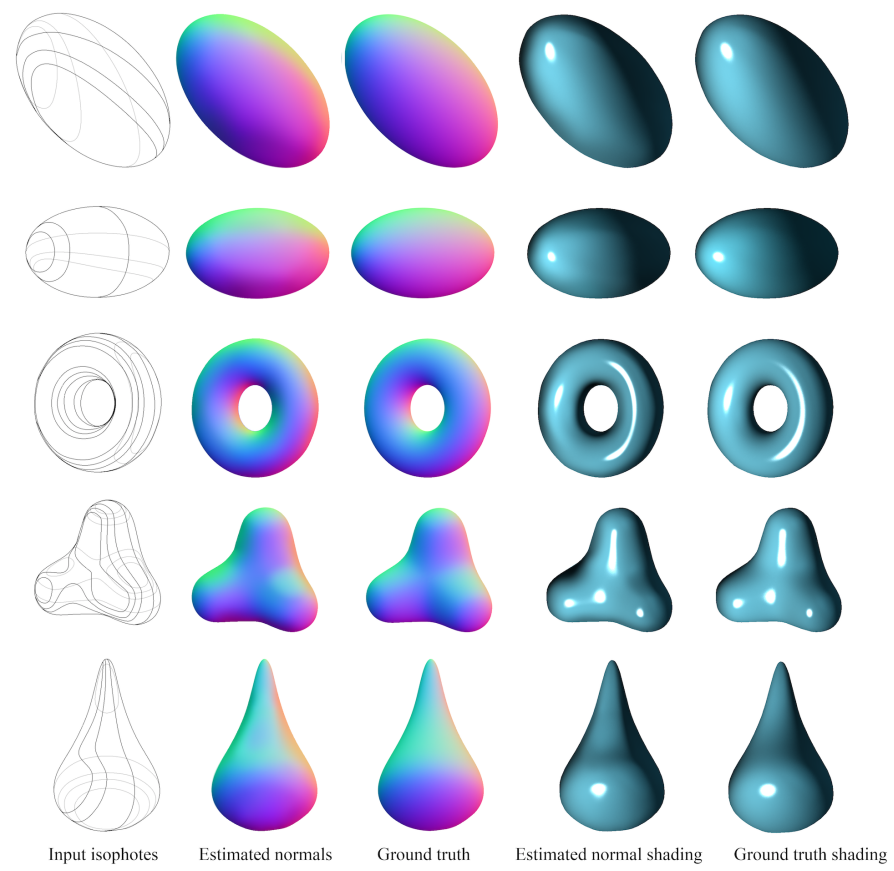

Figure 13: Smooth shading for simple shapes computed with our approach.
${ }_{505}$ User testers. We created a variety of smooth shaded objects 506 with our interface, from simple (Figure 13) to complex (Fig507 ures 2, 14, and 17). The results were produced by an author and 508 two experienced artists (non-authors) (Phone, Purse, Head and ${ }_{509}$ Horse). Results took between 10 minutes (Pear) and 2 hours 510 (Horse) to create (typically 20 minutes), including time spent 511 learning the interface. Users reported being somewhat uncer512 tain about which luminance value to choose for isophotes and ${ }_{513}$ suggested that our interface's "sample illumination sphere" (Fig514 ure 4) be shown with toon shading. Notably, users reported 515 being more confident closer to the 0 luminance value, consistent 516 with our perceptual study.

517 Importantly, our interface is predictable. Adding (or remov518 ing) a single isophote is a stable operation that incrementally 519 affects the resulting normal field and generally produces the 520 desired result (Figure 10). Simple diffusion from silhouettes as 521 in Lumo [10] does not provide designers with sufficient control 522 to model complex and structured objects (Figure 17d). For even ${ }_{523}$ finer control, designers can manually specify normals, such as 524 on the Bunny's belly, or the Head's eye socket (Figure 17).

${ }_{525}$ User testers provided several suggestions to facilitate more 526 accurate user input. Our interface could provide a better visual527 ization of discrete tone-shading by displaying the light-sphere 528 widget with toon shading instead of Lambertian shading; and, 529 when drawing isophotes, our interface could show an isophote ${ }_{530}$ on the light-sphere widget with the same brightness as the cur531 rently selected one. To allow users to rapidly iterate on their 532 corrections, our interface could display a low-resolution normal 533 field quickly, and solve for a full resolution normal field less fre${ }_{534}$ quently. Finally, we could give users control over weights in the ${ }_{535}$ diffusion step of our algorithm, via a marking tool to highlight ${ }_{536}$ important portions of isophotes.

${ }_{537}$ We evaluate our approach for creating height fields from nor- 

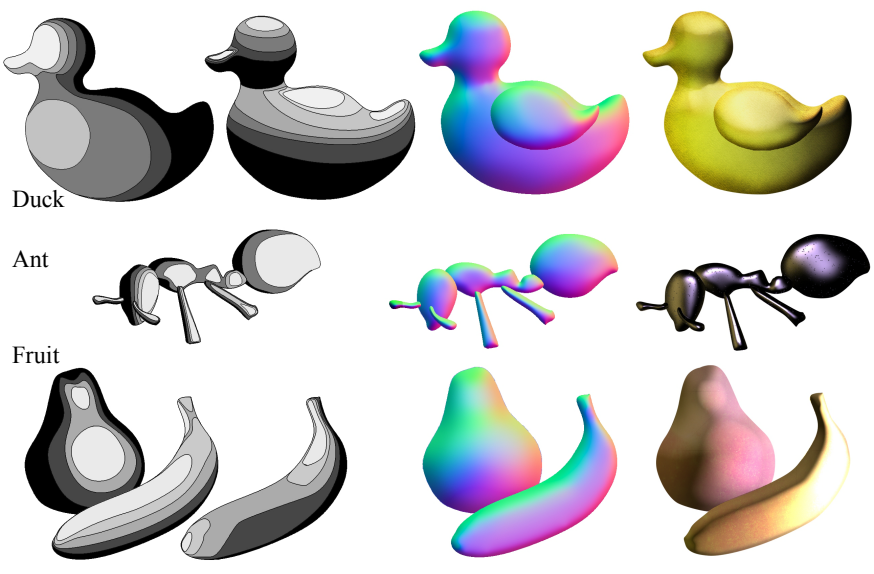

Phone

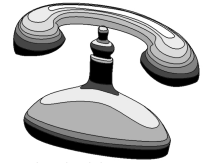

(a) Toon-shaded input

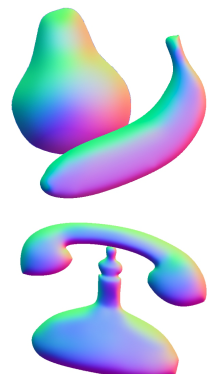

(b) 3D normal field

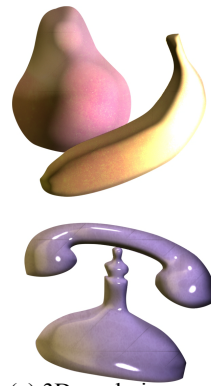

(c) 3D rendering
Figure 14: Artist creations using our interface.

538 mal fields and cast shadows (Section 6) on ground truth data and ${ }_{539}$ artist drawings. Figure 15 displays normal fields, ground truth 540 shadows cast onto a $z=0$ plane, and height fields for known ${ }_{541}$ 3D shapes, as well as height fields resulting from our lifting 542 algorithm. These height maps do not employ our denoising algo${ }_{543}$ rithm. Our height fields are qualitatively and quantitatively quite 544 similar to the ground truth height fields. Note that the ground 545 truth height fields obtained from Maya have been normalized 546 to lie between 0 and 1 . We align height fields generated by our 547 lifting algorithm with ground truth by scaling and offsetting in 548 depth to minimize the sum of squared differences. Note that due 549 to the bas-relief ambiguity [38], height fields are ambiguous up 550 to a uniform scale in the $z$ (depth) direction. The torus illustrates 551 a limitation of our approach. Our algorithm requires that shadow 552 boundaries are visible. However, the shadow boundaries for the 553 slices of the left side of the torus near the top and bottom of the 554 hole are occluded, so an erroneous shadow boundary is detected. ${ }_{555}$ This results in incorrect, discontinuous depth.

556 Figure 16 displays height fields obtained from artist-created 557 normal fields in Figures 2 and 17 augmented with drawn cast 558 shadows. In this setting, our denoising algorithm produces a 559 significant increase in height field quality.

\section{9. Conclusion}

561 For a variety of shapes, smooth 3D normal fields can be 562 quickly created from a toon shaded, or discrete tone image, or 563 even de novo. With the addition of a cast shadow, smooth height ${ }_{564}$ fields can be quickly obtained. Drawing sparse isophotes is 565 far easier than meticulously drawing a smoothly shaded image 566 or "drawing" a smooth, dense 3D normal field. We thus pro${ }_{567}$ pose the novel problem of inverse toon shading and present a ${ }_{568}$ plausible algorithm. Every stage of our algorithm is important: ${ }_{569}$ diffuse-and-project alone produces smooth but high frequency 570 artifacts along isophotes. 2D arc-length-based interpolation
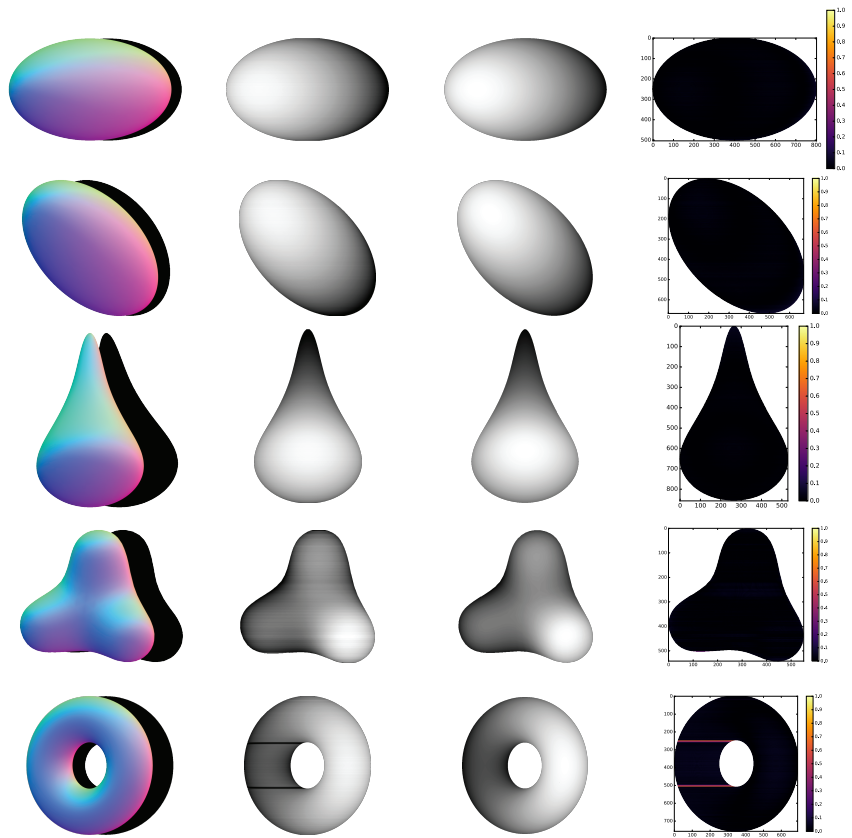

Ground truth

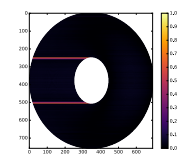

Error

Figure 15: Height fields lifted from ground truth normal maps and shadows. Ground truth height fields are normalized within the range $[0,1]$. Estimated height fields are scaled and offset in depth to align with ground truth height fields (minimizing the sum of squared differences). The error plots the absolute difference.

571 is in accordance with the principle of minimum variation, but 572 does not account for foreshortening, that is handled by itera573 tive 3D refinement, providing a reliable set of surface normals 574 to diffuse-and-project. Our height field algorithm resolves the 575 depth placement resulting from naive integration. The 3D nor576 mal fields we generate allows designers to layer shapes like the 577 Head in Figure 17, and experiment with color, materials and 578 lighting when creating presentation renderings.

579 Our work has several limitations that we hope to address in 580 future work. In addition to the various simplifying assumptions ${ }_{581}$ made in Section 3, our proposed 2D arc-length-based interpo582 lation along isophotes requires that isophotes intersect the sil${ }_{583}$ houette or other isophotes. Certain single-light isophotes, such 584 as on a severely tilted ellipse (Figure 9, second row), are poorly 585 approximated. Our isophote normal estimation algorithms some586 times require manual correction (red gauge in Figure 4). Our ${ }_{587}$ algorithms assume that visible surfaces are $C^{1}$ continuous. There 588 can be no $C^{0}$ edges or occluding contours. For example, the 589 Horse created using our approach (Figure 2) could not be created 590 from the discrete hatched sketch in Figure 1, because the chin 591 forms an occluding contour. We find that some people do not 592 see isophotes well, even though sketching isophotes is important 593 to drawing instruction [2]. Our height field algorithm assumes 594 that shadow boundaries are visible, yet this assumption may be 595 violated by holes or concave regions in the shape.

596 Despite these limitations, we believe that inverse toon shad597 ing, like cross-section drawing, can be a powerful technique to 598 bridge the gap between rapid 2D sketches and 3D presentation 599 renderings. 

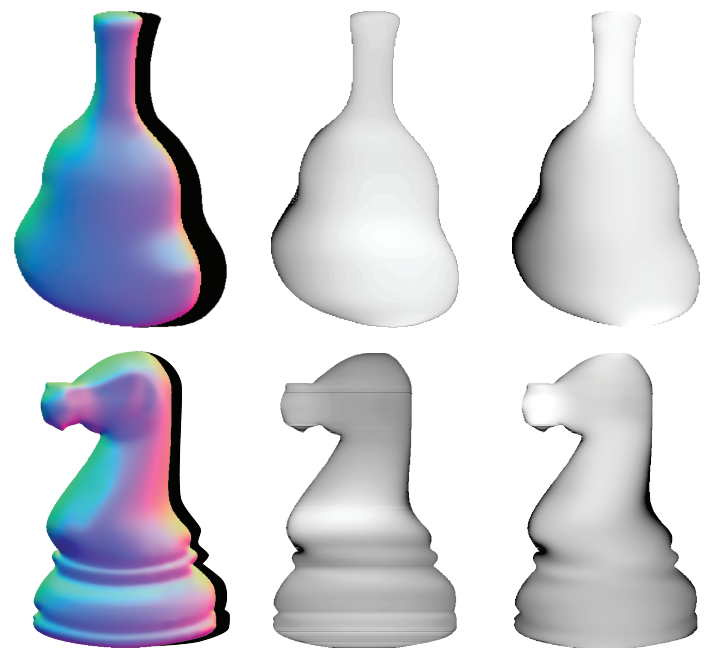

Normal maps with user-drawn shadows

Estimated height fields before denoising

Estimated height field after denoising

Figure 16: Height fields lifted from normal maps and user-drawn shadows.

\section{Acknowledgements}

601 We are grateful to Adrien Bousseau and the anonymous re602 viewers for their constructive comments. This work was sup${ }_{603}$ ported in part by the United States National Science Foundation 604 (IIS-1451198 and IIS-1453018) and a Google research award.

05

] A. L. Guptill, S. E. Meyer, Rendering in Pen and Ink: The Classic Book on Pen-And-Ink Techniques for Artists, Illustrators, Architects, and Designers, Watson-Guptill, 1997.

8 [2] B. Edwards, The New Drawing on the Right Side of the Brain, Jeremy P. Tarcher/Putnam, ISBN 9780874774245, URL http://books .google.ca/books?id=MKD7NG267mIC, 1999.

[3] K. Eissen, R. Steur, Sketching: Drawing Techniques for Product Designers, Bis Publishers, 2008.

3 [4] D. Powell, Presentation techniques: a guide to drawing and presenting design ideas, Little, Brown, ISBN 9780316912433, URL http://books.google.co.in/books?id=CAiAQgAACAAJ, 1994.

[5] C. Shao, A. Bousseau, A. Sheffer, K. Singh, CrossShade: Shading Concept Sketches Using Cross-Section Curves, ACM Transactions on Graphics (Proc. SIGGRAPH) 31 (4).

[6] Q. Xu, Y. Gingold, K. Singh, Inverse Toon Shading: Interactive Normal Field Modeling with Isophotes, in: Proceedings of Sketch-Based Interfaces and Modeling (SBIM), 2015.

[7] D. Sýkora, J. Dingliana, S. Collins, LazyBrush: Flexible Painting Tool for Hand-drawn Cartoons, Computer Graphics Forum (Proc. EUROGRAPHICS) 28 (2).

[8] A. Orzan, A. Bousseau, H. Winnemöller, P. Barla, J. Thollot, D. Salesin, Diffusion Curves: A Vector Representation for Smooth-Shaded Images, ACM Trans. on Graph. (Proc. SIGGRAPH) 27.

[9] M. Finch, J. Snyder, H. Hoppe, Freeform vector graphics with controlled thin-plate splines, ACM Trans. on Graph. (Proc. SIGGRAPH Asia) 30.

630 [10] S. F. Johnston, Lumo: illumination for cel animation, in: Proc. Symp. on Non-Photorealistic Animation and Rendering, 2002.

632 [11] H. Winnemöller, A. Orzan, L. Boissieux, J. Thollot, Texture Design and 633 Draping in 2D Images, Computer Graphics Forum (Proc. Symp. on Rendering) 28 (4).

635 [12] D. Sýkora, L. Kavan, M. Čadík, O. Jamriška, A. Jacobson, B. Whited M. Simmons, O. Sorkine-Hornung, Ink-and-ray: Bas-relief Meshes for Adding Global Illumination Effects to Hand-drawn Characters, ACM Trans. Graph. 33 (2) (2014) 16:1-16:15, ISSN 0730-0301, doi:10.1145/2591011, URL http://doi.acm.org/10.1145/2591011.

40 [13] L. Zhang, G. Dugas-Phocion, J.-S. Samson, S. M. Seitz, Single View Modeling of Free-Form Scenes, in: IEEE Computer Vision and Pattern Recognition, 990-997, 2002.
643 [14] M. Okabe, G. Zeng, Y. Matsushita, T. Igarashi, L. Quan, H. yeung Shum, Single-view relighting with normal map painting, in: Proc. Pacific Graphics, 27-34, 2006.

6 [15] T.-P. Wu, C.-K. Tang, M. S. Brown, H.-Y. Shum, ShapePalettes: interactive normal transfer via sketching, ACM Trans. on Graph. (Proc. of SIGGRAPH) 26, ISSN 0730-0301.

9 [16] J.-D. Durou, M. Falcone, M. Sagona, Numerical methods for shape-from-shading: A new survey with benchmarks, Computer Vision and Image Understanding 109 (2008) 2243, ISSN 1077-3142, doi:10.1016/j.cviu.2007.09.003, URL http: //portal.acm.org/citation. cfm?id=1326363.1326482.

[17] R. J. Woodham, Photometric method for determining surface orientation from multiple images, Optical Engineering 19 (1) (1980) 191139-191139, doi:10.1117/12.7972479, URL http://dx.doi.org/10.1117/12.7972479.

58 [18] B. Xu, W. Chang, A. Sheffer, A. Bousseau, J. McCrae, K. Singh, True2Form: 3D Curve Networks from 2D Sketches via Selective Regularization, Transactions on Graphics (Proc. SIGGRAPH) 33 (4), doi: 2601097.2601128.

[19] P. Joshi, N. A. Carr, Repoussé: Automatic Inflation of 2D Artwork, in: Proc. of Sketch Based Interfaces and Modeling, 2008.

664 [20] D. Cremers, Fast and Globally Optimal Single View Reconstruction of Curved Objects, in: Computer Vision and Pattern Recognition, CVPR, ISBN 978-1-4673-1226-4, 534-541, URL http: //dl . acm.org/citation. cfm?id=2354409. 2354928, 2012.

8 [21] T. Igarashi, S. Matsuoka, H. Tanaka, Teddy: a sketching interface for 3D freeform design, SIGGRAPH

[22] A. Ecker, K. Kutulakos, A. Jepson, Shape from Planar Curves: A Linear Escape from Flatland, in: IEEE Computer Vision and Pattern Recognition, doi:10.1109/CVPR.2007.383020, 2007.

[23] P. Tan, T. Zickler, A projective framework for radiometric image analysis, in: Computer Vision and Pattern Recognition, CVPR, IEEE, 2977-2984, 2009.

6 [24] M. Kaplan, E. Cohen, Producing Models from Drawings of Curved Surfaces, in: Sketch-Based Interfaces and Modeling, SBM, ISBN 3-905673-39-8, 51-59, doi:10.2312/SBM/SBM06/051-058, URL http://dx.doi.org/10.2312/SBM/SBM06/051-058, 2006.

[25] Y. Gingold, T. Igarashi, D. Zorin, Structured Annotations for 2D-to-3D Modeling, ACM Trans. on Graph. (Proc. SIGGRAPH Asia) 28 (5), ISSN 0730-0301.

[26] Y. Gingold, D. Zorin, Shading-based surface editing, Transactions on Graphics (Proc. SIGGRAPH) 27 (3) (2008) 95:1-95:9, ISSN 0730-0301.

[27] H. Theisel, Are Isophotes and Reflection Lines the Same?, Comput. Aided Geom. Des. 18 (7) (2001) 711-722, ISSN 0167-8396, doi:10.1016/S0167-8396(01)00063-2, http://dx.doi .org/10.1016/S0167-8396(01) 00063-2.

9 [28] T. Poeschl, Detecting Surface Irregularities Using Isophotes, Comput. Aided Geom. Des. 1 (2) (1984) 163-168, ISSN 0167-8396, doi:10.1016/0167-8396(84)90028-1, URL http://dx.doi .org/10.1016/0167-8396(84)90028-1.

[29] A. Deslandes, D. L. Bonner, Reflection line control, uS Patent 6,717,579, 2004.

5 [30] J. Loos, G. Greiner, H.-P. Seidel, Modeling of Surfaces with Fair Reflection Line Pattern, in: Shape Modeling and Applications, SMI, ISBN 0-7695-0065-X, 256, URL http://dl.acm.org/citation. cfm?id=829509.830285, 1999.

9 [31] E. Tosun, Y. Gingold, J. Reisman, D. Zorin, Shape optimization using reflection lines, in: Eurographics Symposium on Geometry Processing, SGP, ISBN 978-3-905673-46-3, 193-202, 2007.

[32] B. Gooch, A. Gooch, Non-Photorealistic Rendering, AK Peters Ltd, iSBN: 1-56881-133-0, 2001

[33] T. Goodwin, I. Vollick, A. Hertzmann, Isophote Distance: A Shading Approach to Artistic Stroke Thickness, in: Proceedings of Non-photorealistic Animation and Rendering, NPAR, ISBN 978-1-59593-624-0, 53-62, doi:10.1145/1274871.1274880, URL http://doi.acm.org/10.1145/1274871.1274880, 2007.

[34] J. J. Koenderink, A. J. V. Doorn, A. M. L. Kappers, Surface perception in pictures, Perception \& Psychophysics (1992) 487-496.

1 [35] J. McCrae, K. Singh, Sketching piecewise clothoid curves, Computers \& Graphics 33 (4) (2009) 452-461.

713 [36] R. Halîr, J. Flusser, Numerically stable direct least squares fitting of el- 

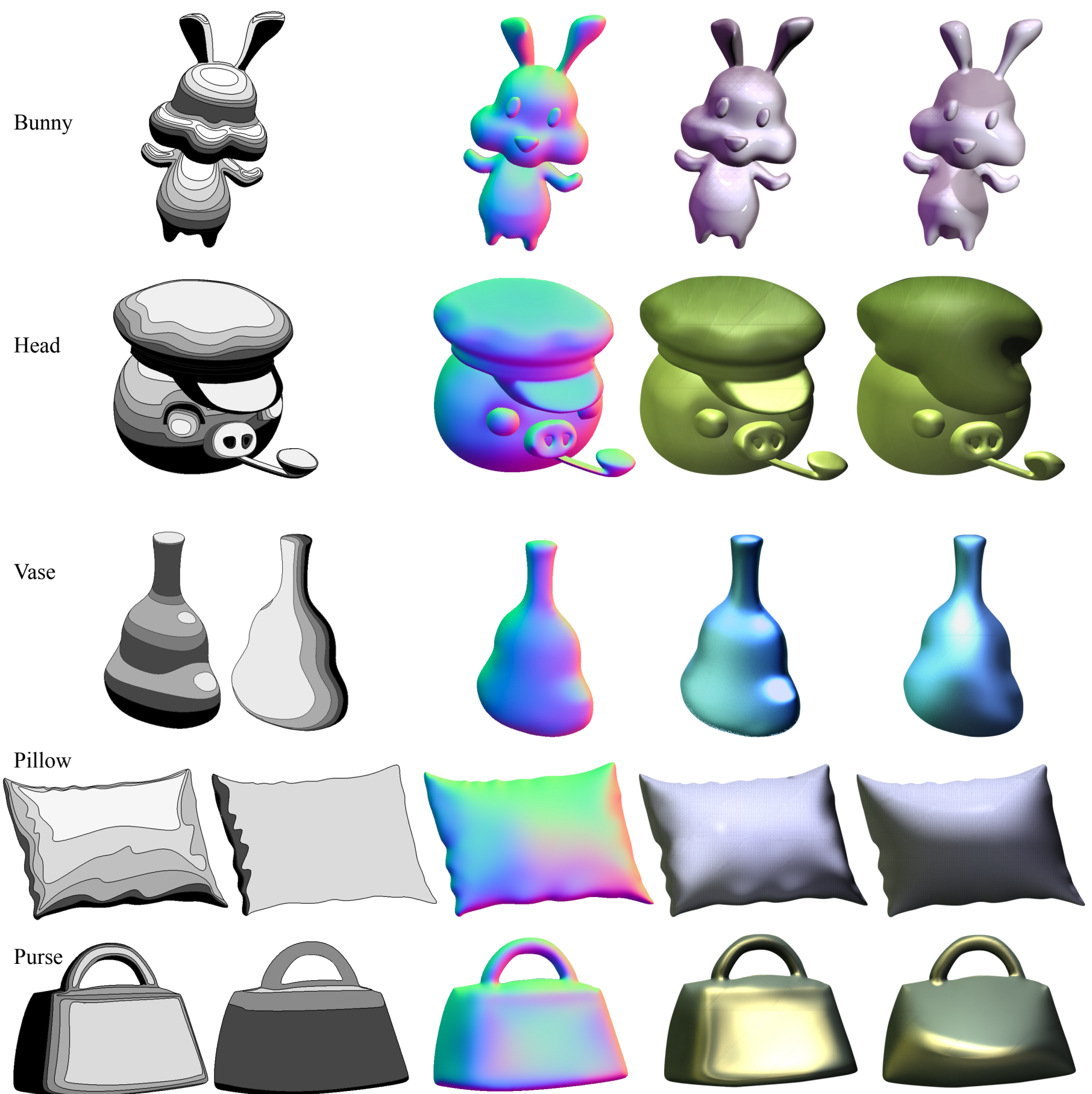

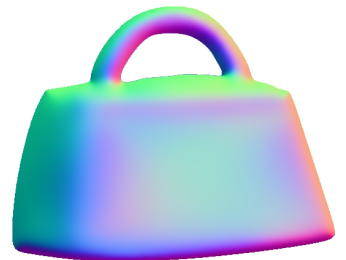

(b) 3D normal field

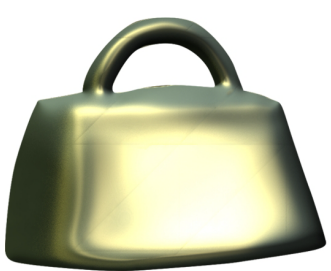

(c) $3 \mathrm{D}$ rendering

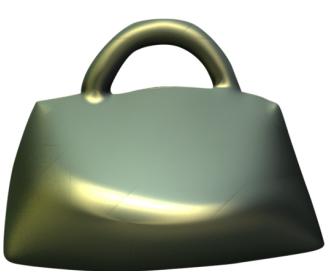

(d) Silhouette diffusion

Figure 17: Artist creations using our interface. Artists created toon-shaded inputs from one or two light directions (a). The isophotes between discrete shading levels were input into our system to obtain a 3D normal field (b). 3D renderings of our results (c) show the control and detail of isophotes over simple silhouette diffusion from $(d)$. 
lipses, in: 6th International Conference in Central Europe on Computer Graphics and Visualization (WSCG), vol. 98, 125-132, 1998.

716 [37] P. Company, R. Plumed, P. A. Varley, A fast approach for perceptuallybased fitting strokes into elliptical arcs, The Visual Computer 31 (6) (2015) 775-785, ISSN 0178-2789, doi:10.1007/s00371-015-1099-6, URL http://dx.doi.org/10.1007/s00371-015-1099-6.

20 [38] P. N. Belhumeur, D. J. Kriegman, A. L. Yuille, The Bas-Relief Ambiguity, in: Proceedings of IEEE CVPR, 1060-1066, 1997. 